\title{
LA APLICACIÓN DEL APRENDIZAJE COLABORATIVO Y EL RESULTADO SEGÚN LOS ENFOQUES DE APRENDIZAJE
}

\section{THE USE OF THE COLLABORATIVE LEARNING AND THE RESULT ACCORDING TO THE APPROACHES OF LEARNING}

María Pilar Flores Asenjo'. Universidad Católica San Antonio de Murcia. España. pflores@ucam.edu

Miguel Ángel Beltrán Bueno². Universidad Católica San Antonio de Murcia. España. mabeltran@ucam.edu

\section{RESUMEN}

Las técnicas de aprendizaje colaborativo son frecuentemente utilizadas como metodología para favorecer el aprendizaje en grupo y el aprendizaje con enfoque profundo. Para conseguir estos propósitos, el docente-instructor debe realizar una adecuada planificación y favorecer un adecuado clima de trabajo. El aprendizaje profundo se caracteriza por un interés intrínseco por la materia y la motivación extrínseca o superficial, en cambio, supone el uso pasivo de la información; por tanto, para los docentes podría ser adecuado crear los grupos y organizar el trabajo en función de sus diferentes características.

PALABRAS CLAVE: aprendizaje colaborativo, motivación superficial, motivación profunda, clima de trabajo, factores de estrés.

\section{ABSTRACT}

The collaborative activities should facilitate ways for group members to learn from each other and formal ways of structuring activities in a learning environment that includes specific elements intended to increase the potential for deep learning by the participants. The instructor should maintain a certain degree of control in managing and guiding the group so that the learning outcomes were achieved. Deep learning is marked by deliberate intention to learn, as well as individual and social orientations to learning however superficial learning is oriented to information-reproducing work. Educational planners should ensure an adequate mix of students with different learning motivations in the collaborative groups to achieve desired objectives.

KEY WORDS: collaborative learning, superficial motivation, deep motivation.

\footnotetext{
1 María del Pilar Flores-Asenjo: Universidad Católica de Murcia. Administración de Empresas. Murcia, España.

${ }^{2}$ Miguel Ángel Beltrán Bueno: Universidad Católica de Murcia. Turismo. Murcia, España.
} 


\section{Cómo citar el artículo:}

Flores Asenjo, M. P. y Beltrán Bueno, M. A. (2015). La aplicación del aprendizaje colaborativo y el resultado según los enfoques de aprendizaje. Revista de Ciencias de la Comunicación e Información, 20, 45-53.

DOI: http://doi.org/10.35742/revistacccomunicacioneinformacion.2015.20.45-53

\section{INTRODUCCIÓN}

\subsection{Aprendizaje colaborativo}

Uno de los objetivos para los docentes que más explícitamente se destaca en el nuevo marco formativo es el de enseñar a aprender, lo que pone de relieve la necesidad de adoptar metodologías docentes que generen mayor motivación (Arquero y Jiménez, 1999). Tal y como establece Jonassen (1994), las características generales del aprendizaje cooperativo y colaborativo son enfatizar la construcción del conocimiento en vez de la reproducción del mismo, fomentar la reflexión basada en la experiencia, y apoyar la construcción colaborativa del conocimiento a través de la negociación social y no de la competencia. Por este motivo, las investigaciones más recientes dan cada vez mayor importancia a los procesos de trabajo grupal (Cabero y López, 2009; Romero de Ávila, 2010).

La motivación, la capacidad de abordar problemas complejos, la participación activa y la implicación por medio de la distribución de roles son algunos de los rasgos que definen los beneficios de las técnicas de aprendizaje colaborativo (Gértrudix, 2006). La colaboración fomenta que los estudiantes se sientan parte de una comunidad de aprendizaje, lo que favorece la construcción de conocimiento compartido y crea un espíritu comunitario (Garrison y Anderson, 2005; Tu, 2004). Los alumnos interiorizan actitudes básicas necesarias para un trabajo en grupo, como el compromiso, la transparencia, la constancia y el respeto. Aprenden de su propia experiencia, reforzando el aspecto motivador de esta metodología que fomenta la discusión, la defensa de argumentos y el acercamiento entre posturas.

Pero todas estas virtudes del método requieren realizar una adecuada planificación donde se definan los objetivos a alcanzar, los contenidos a tratar y la metodología a aplicar para su desarrollo (Echazarreta et al., 2009). El docente debe estar presente en cada uno de los pasos del alumno como orientador y guía de la experiencia de aprendizaje; una supervisión constante del profesor, que suscite un clima adecuado de cooperación, y una eficaz programación y organización de la dinámica. que minimice las dificultades principales en el uso de estas metodologías: evitar el desequilibrio en la participación y los conflictos que puede generar, motivar el trabajo en equipo de alumnos, preparar la documentación adecuada a este tipo de aprendizaje y encontrar una forma correcta de evaluación de los resultados. La programación y organización de las tareas deben de tener en cuenta también otros factores estresantes del clima de trabajo en equipo como la percepción de sobrecarga de trabajo y la presión temporal (Salanova et al., 2005).

Los estudios sobre aprendizaje en equipo y generación de conocimiento colectivo sugieren que cuando se comparten objetivos el aprendizaje de equipo es mayor (De 
Dreu, 2007; Rico et al, 2010). Crear un adecuado clima de equipo, donde se compartan visiones, procedimientos y objetivos, y donde se incentive la participación, es fundamental para el logro del aprendizaje colectivo (Lowen y Loo, 2004).

Los problemas de interrelaciones personales de los miembros del grupo pueden interferir en el logro de los objetivos al provocar una colaboración no equitativa de los mismos (Oakley et al., 2004) y, por tanto, conflictos que reducen las virtudes del trabajo conjunto. La eficiencia de los aprendizajes en grupo dependen del grado de interdependencia positiva generada entre el grupo, pero también del reconocimiento individual de responsabilidad de los participantes (Johnson y Johnson, 1998). El fenómeno "free rider" ("parásito") en el trabajo colaborativo puede provocar el fracaso de la metodología y es uno de los aspectos a tener en cuenta en el papel de moderador del docente; por este motivo, la metodología debe de incorporar mecanismos que minimicen la posibilidad de aparición de estas actitudes negativas a través de sistemas tanto de evaluación conjunta como individual del trabajo.

El cambio en el modelo de metodologías docentes implica generalmente un cambio en la planificación y ejecución de la docencia, pero también debería llevar asociado un cambio en la evaluación (Rué, 2008; p. 3). La literatura de Psicología ha destacado que la identidad social y el compromiso de los miembros del equipo con su grupo influyen positivamente en el rendimiento del equipo (Haslam et al., 2006), pero se ha de poner especial cuidado en la elección del sistema de evaluación, que ha de ser comprendido correctamente por los alumnos, si se desea motivar para el logro de los mejores resultados.

\subsection{Motivación profunda y superficial del estudiante y aprendizaje colaborativo}

Según Marton y Säljö (1976), una motivación profunda implica un interés intrínseco por la materia y el deseo de lograr que el aprendizaje tenga significación personal (intención de comprender y relacionar conocimientos). La motivación extrínseca o superficial, en cambio, supone el uso pasivo de la información (aceptación de las ideas) y suelen estar ligadas a las estrategias superficiales de carácter memorístico y repetitivo (focalización), sin implicación del alumno en el aprendizaje.

El enfoque de aprendizaje describe la naturaleza de la relación existente entre el alumno, el contexto y la tarea (Biggs et al., 2001; p. 137), por tanto, no es estable e inalterable sino que depende de las metodologías de enseñanza utilizadas y de su evaluación. De esta manera, metodologías expositivas y con evaluaciones memorísticas se relacionarían más con posicionamientos superficiales de los estudiantes, mientras que las que se centran en el autoaprendizaje, la diversidad de métodos y evaluaciones, requerirían una mayor profundidad de enfoque (Argos et al., 2013; p. 183).

\section{OBJETIVOS}

Atendiendo a esta clasificación y definición, parece justificado que la forma de trabajar en equipo de aquellos que tienen una motivación profunda y los que tienen una motivación superficial sea diferente, ya que los objetivos y procedimientos también lo son. Además, la manera de enfrentarse a los problemas de clima de 
trabajo en equipo y de afrontar los factores estresantes (sobrecarga y presión temporal) también puede diferir. En ese caso, el conocimiento de perfil de motivación de los estudiantes facilitaría al docente el trabajo de organización y planificación en el uso de metodología colaborativa, al predecir cómo afrontarán el trabajo los estudiantes y cuáles serían los principales factores de ineficacia de los grupos.

\section{METODOLOGÍA}

En este estudio se pretende observar la forma de trabajar en equipo en función del tipo de motivación de aprendizaje del alumno. Para ello, primero se clasificó al alumno según su motivación utilizando una versión reducida del cuestionario de proceso de estudio de dos factores (R-CPE-2F) validado por Saucedo y Almenara (2005) para universitarios españoles; a continuación, a los grupos de 3 componentes homogéneos se les planteó un "caso-problema" $y$, finalmente, se realizó una encuesta sobre interacción grupal basada en el autoinforme de interacción grupal (AIG) de Sáiz y Gómez (2007), que consta de 30 ítems con 5 posibles respuestas tipo Likert sobre metodología del trabajo en grupo, formas de colaboración, percepción de las aportaciones, clima de trabajo y resultados, y una encuesta sobre factores estresantes del clima de trabajo formada por 6 ítems sobre sobrecarga de trabajo, problemas de "parásito" y tiempo para las tareas.

\section{RESULTADOS}

El "caso-problema" fue planteado a 42 alumnos de segundo curso de Administración y Dirección de empresas con una edad media de 19,7 años $( \pm 3,3)$, 18 hombres y 24 mujeres. Para poder formar grupos homogéneos, cada cuestionario de motivación tenía un número identificador o clave que sólo conocía el alumno. En un listado se informó a los alumnos de los grupos que se habían realizado atendiendo a su clasificación según motivación: se formaron 14 grupos homogéneos, 5 con motivaciones mayoritariamente profundas de sus componentes y 9 con motivaciones superficiales.

El cuestionario de proceso de estudio de dos factores dio como resultado 26 alumnos con motivación superficial y 16 con motivación profunda, por lo que se formaron 5 grupos con motivación mayoritariamente profunda y 9 con motivación superficial.

Para poder confirmar las dimensiones y subdimensiones del AIG, se ha utilizado el análisis factorial como técnica analítica multivariada que nos permitiré reducir el conjunto de los 30 ítems propuestos a unos pocos factores o dimensiones. Dentro de las posibilidades que permite este análisis se optó por aplicar el análisis de los componentes principales (ACP), por ser el más apropiado para el estudio de escalas cuya estructura ya ha sido objeto de análisis previos. Previamente al análisis factorial se confirmó que las condiciones de las variables eran apropiadas para este tipo de análisis, mediante el cálculo del determinante de correlación, la prueba KMO (Kaiser-Meyer-Olkin) y el test de esfericidad de Barlett.

De la varianza total explicada se seleccionaron los cinco primeros factores, que acumulan el $73,09 \%$ de la varianza total. El análisis factorial de las respuestas a los 30 
ítems del AIG dio lugar a las siguientes dimensiones: $(F 1)$ métodos o procedimientos de trabajo del grupo ("Cuando una explicación no satisfacía a los miembros del grupo se han buscado explicaciones alternativas", "Las normas de funcionamiento del grupo fueron fijadas por adelantado para permitirnos evaluar el funcionamiento del grupo mientras trabajábamos", "Las tareas han requerido que nos reunamos y trabajemos juntos; nunca hemos trabajado solos para combinar nuestro trabajo al final"), (F2) nivel de cooperación y clima del grupo ("Los miembros del grupo han participado en la construcción de los argumentos de los otros miembros del grupo", "Planteamos las discusiones sobre la base de la información discutida en grupo", "Todos los miembros han trabajado de forma equitativa", "Mientras el trabajo ha ido progresando el grupo se ha cohesionado más", "Cuando alguien rebatió a un miembro del grupo lo hizo argumentando su posición"), (F3) idoneidad del grupo ("La mayoría de los miembros del grupo han valorado positivamente trabajar en forma grupal", "El grupo en el que he trabajado tenía el tamaño ideal"), (F4) gestión de conflictos ("Uno o más miembros del grupo fueron contradichos por los demás miembros", "Mi grupo ha funcionado de forma excelente") y (F5) nivel de percepción de aportación individual al grupo ("Los otros miembros del grupo conocían lo que yo estaba haciendo, podían comprobar mi trabajo con facilidad y hacer un seguimiento de mis aportaciones", "Mi contribución individual ha contribuido al resultado final del trabajo").

Todas las intercorrelaciones entre dimensiones fueron positivas y significativas, lo que parece evidenciar que todas las dimensiones miden el mismo constructo; y el análisis de las correlaciones de cada dimensión nos determinó que los valores obtenidos presentan una significación estadística (1\%).

En cuanto a los ítems, se realizó, por un lado, el cálculo del grado de relación existente entre ellos y el total de su dimensión así como con el total de la escala, y por otro lado, se determinó el Alpha de Cronbach, que es uno de los más potentes indicadores para medir la consistencia interna de un instrumento. El Alpha de Cronbrach obtenido de toda la escala fue de 0,895 , coeficiente que confirma que todas las puntuaciones proporcionadas por la escala son fiables. El criterio para determinar la consistencia interna de cada ítem fue el coeficiente de discriminación (correlación) igual o inferior a 0,33, considerando que los ítems cuya correlación respecto a la totalidad de la escala son iguales o inferiores a esta cifra, al ser suprimidos, aumentan el coeficiente alpha de la totalidad de la escala. Además, el análisis de la consistencia por dimensiones ofreció unos índices de fiabilidad superiores a 0,7.

Gráfico 1. Puntuación de los factores de interacción grupal.

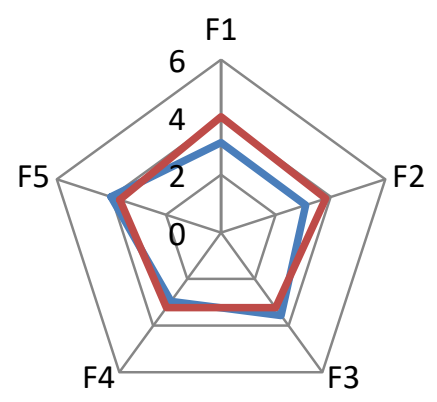

SUPERFICIAL

$\longrightarrow$ PROFUNDA

Fuente: Elaboración propia. 
Una vez asegurada la fiabilidad de la escala, se analizó para los grupos de motivación superficial y profunda. Como puede apreciarse en el gráfico 1, la media de puntuación de los factores para los grupos de motivación profunda es superior que la de los grupos de motivación superficial, salvo en los factores F3 (Idoneidad del grupo) y F5 (Nivel de percepción de aportación individual al grupo).

En cuanto a las preguntas referidas a los factores estresantes del clima de trabajo, el gráfico 2 muestra una importante diferencia entre las respuestas de los dos grupos (estadísticamente significativa según Test-T), salvo en las preguntas referidas al tiempo como factor estresante (P1. "La falta de tiempo ha sido un factor de conflicto en el desarrollo del trabajo" y P2. "El tiempo programado para las actividades NO ha sido el suficiente para un buen desarrollo"). Aquellos que tienen una motivación superficial encuentran más estresante la sobrecarga de trabajo (P5 y P6) y perciben mayor efecto "parásito" en los grupos (P3 y P4).

Gráfico 2. Respuestas a factores estresantes.

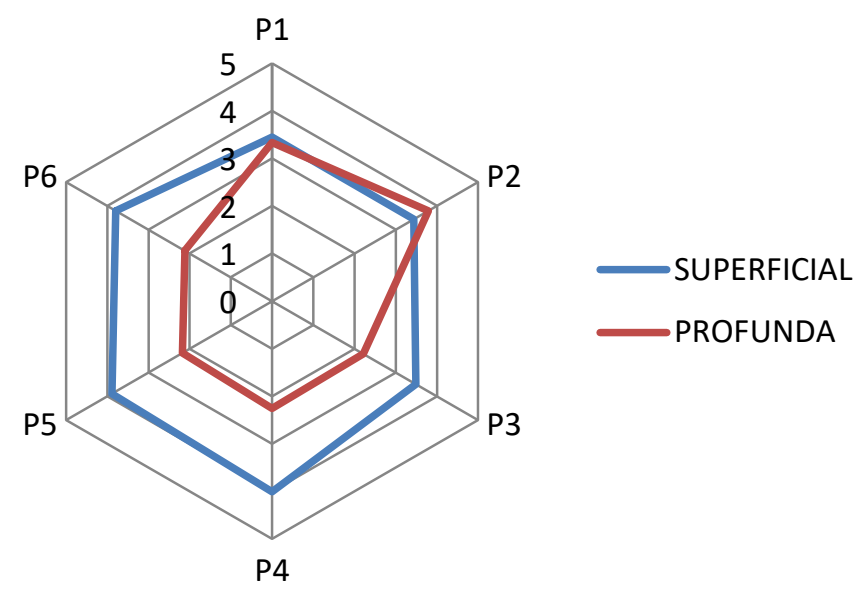

Fuente: Elaboración propia.

\section{CONCLUSIONES Y DISCUSIÓN}

Los grupos compuestos por estudiantes con motivación profunda han percibido en el desarrollo del caso que su organización del trabajo y su nivel de cooperación han sido los factores determinantes de su resultado, siendo el tiempo el único factor estresante de su clima de trabajo. En cambio, los grupos formados por estudiantes con motivación superficial estiman que el resultado del grupo se debe a su aportación personal y el clima trabajo se ve perjudicado por la sobrecarga de trabajo y por el efecto "parásito".

Parece por tanto que, para esta muestra, la interacción grupal ha sido más positiva para los alumnos con motivación profunda al organizarse mejor y crear un mejor clima, aunque su interés en comprender y relacionar conceptos les ha llevado a tener mayores limitaciones de tiempo que las deseadas.

Los grupos formados por estudiantes con motivación superficial, con estrategias de aprendizaje memorístico y repetitivo e implicación pasiva en el aprendizaje, son los 
que mayores problemas de percepción de factores estresantes de clima de trabajo grupal. Además, su forma de enfocar los objetivos de aprendizaje y organizase han creado mayores problemas de efecto "parásito".

Tal y como se ha indicado, el docente debe estar presente en cada uno de los pasos del alumno como orientador y guía de la experiencia de aprendizaje; supervisando y suscitando un clima adecuado de cooperación. El estudio de la motivación de aprendizaje de los alumnos podría entonces servir al docente para prever los principales problemas que pueden surgir en el desarrollo de los casos y organizar mejor las sesiones y los grupos teniendo en cuenta sus particularidades.

\section{REFERENCIAS}

\section{Libros:}

Cabero, J. y López, E. (2009). Evaluación de materiales multimedia en red en el Espacio Europeo de Educación Superior [EEES]. Colección Redes № 17. Barcelona: Ed. Davinci.

Garrison, D. R. y Anderson, T. (2005) El e-learning en el siglo XXI. Barcelona: Octaedro.

Tu, C. (2004). Online collaborative learning communities: Twenty-one designs to building an online collaborative learning community. Westport, CT: Libraries Unlimited.

\section{Artículos en revistas:}

Arquero, J. L. y Jiménez, S. M. (1999). Influencia del estudio de casos en la mejora del aprendizaje, adquisición de capacidades no técnicas y motivación en análisis contable. Revista de Enseñanza Universitaria Extraordinario, 1, 225-242.

Biggs, J. B., Kember, D. y Leung, D. (2001). The revised two-factor Study Process Questionnaire: R- SPQ-2F. British Journal of Educational Psychology, 71, 133149.

De Dreu, C. K. W. (2007). Cooperative outcome interdependence, task reflexivity and team performance: A motivated information processing perspective. Journal of Applied Psychology, 92, 628-638.

Haslam, S.A., Ryan, M.K., Postmes, T., Spears, R., Jetten, J. \& Webley, P. (2006). Stickingto our guns: Social identity as a basis for the maintenance of commitment tofaltering organizational projects. Journal of Organizational Behavior, 27, 607-628.

Johnson, D. W., Johnson, R. T., \& Smith, K. A. (1998). Cooperative learning returns to college what evidence is there that it works? Change: the magazine of higher learning, 30(4), 26-35. 
Jonassen, D.H. (1994). Thinking technology: toward a constructivist design model. Educational Technology. 34(4), 34-37.

Loewen, P. \& Loo, R. (2004). Assessing team climate by qualitative and quantitative approaches. The Learning Organization, 11(3), 260-272.

Marton, F. y Säljö, R. (1976). On qualitative Differences in Learning: I Outcome and Process, British Journal of Educational Psychology, 46, 4-11.

Oakley, B., Felder, R. M., Brent, R., \& Elhajj, I. (2004). Turning student groups into effective teams. Journal of student centered learning, 2(1), 9-34.

Rico, R., \& Alcover, C. Ma. y Tabernero, C. (2010). Efectividad de los equipos de trabajo, una revisión de la última década de investigación (1999-2009). Revista de Psicología del Trabajo y de las Organizaciones, 26(1), 47-71.

Sáiz, M. S. I., y Gómez, G. R. (2007). El trabajo colaborativo en las aulas universitarias: reflexiones desde la autoevaluación. Revista de educación, 344, 229-230.

Saucedo, M. y Almenara, J.C. (2005). Enfoques de aprendizaje, rendimiento académico y satisfacción de los alumnos en formación de entornos virtuales. Revista píxel-Bit, Revista de Medios y Educación, 25, 93-115.

Salanova, M., Grau, R. M. \& Martínez, I. M. (2005). Demandas laborales y conductas de afrontamiento: el rol modulador de la autoeficacia profesional. Psicothema, 17(3), 390-395.

Recursos electrónicos:

Argos, J., Ezquerra, P., Manuel Osoro, J., Salvador, L., \& Castro, A. (2014). La evaluación de los aprendizajes de los estudiantes en el marco del Espacio Europeo de Educación Superior (EEES): sus prácticas, preferencias y evolución. European Journal of investigation in health, psychology and education, 3(3), 181194. Recuperado de http://ejihpe.es/index.php/journal/article/viewFile/41/pdf

Echazarreta, C; Prados, F.; Poch, J. \& Soler, J. (2009). La competencia El trabajo colaborativo: una oportunidad para incorporar las TIC en la didáctica universitaria. Descripción de la experiencia con la plataforma ACME (UdG). Revista sobre la sociedad del conocimiento, 8. Recuperado de http://www.uoc.edu/uocpapers/8/dt/esp/echazarreta prados poch soler.pdf.

Gértrudix Barrio, M. (2006). Convergencia multimedia y Educación. ICONO, 14 (7). Consultado el 2 de marzo de 2014. Disponible en: http://www.icono14.net/ojs/index.php/icono14/article/viewFile/401/277.

Romero de Ávila Torrijos, D. (2010). El aprendizaje por competencias y su evaluación: una aplicación para la asignatura Macroeconomía de nivel intermedio. @tic.revista d'innovació educativa (4). Recuperado de ojs.uv.es/index.php/attic/article/download/148/1103. 
Rué, J. (2008). Formar en competencias en la universidad: entre la relevancia y la banalidad. Red U. Revista de Docencia Universitaria, 2 (1), 1-19. Recuperado de dialnet.unirioja.es/descarga/articulo/2749773.pd 